\title{
Estimation of Market Risk Premium for Japan
}

\author{
Seoungpil Ahn (Corresponding author) \\ Dept. of Finance, National University of Singapore \\ 1 Business Link, Singapore 117592 \\ Tel: 65-6516-4555 E-mail: bizsa@nus.edu.sg \\ Keshab Shrestha \\ Nanyang Business School, Nanyang Technological University \\ Nanyang Avenue, Singapore 639798 \\ Tel: 65-6790-4642Ｅ-mail: akeshab@ntu.edu.sg
}

\begin{abstract}
In this paper, the time series of risk aversion parameter is estimated for the Japanese stock market using weekly return data covering $2 / 7 / 1973$ to $12 / 27 / 2000$. The time series of risk aversion parameter is estimated with the Time Varying Parameter (EVP) GARCH-M model proposed by Chou, Engle and Kane (1992), which allows for the risk aversion parameter to change over time by modeling the risk aversion parameter to follow a random walk process. The risk aversion parameter is found to range between 3.5 to 2.2. We also find that the risk aversion parameter has not significantly changed over time. This implies that most of the variation in excess return can be explained by the variation in the market (variance) risk.
\end{abstract}

Keywords: GARCH-M, Kalman Filtering, risk aversion, time-varying parameter, volatility. 


\section{Introduction}

Investors' preference to risk is central to financial economic theories. Many economic and financial theories assume that investors are risk averse. This implies that investors demand compensation, in terms of higher expected return compared to the return on the risk-free asset (i.e., a positive risk premium), for taking risk. Furthermore, the investors will demand higher risk premium for the same level of risk if they become more risk averse over time. Therefore, the historical (or time series) characteristics of risk aversion are an important element to understand the time series patterns of return process.

The market price of risk (measured by the risk premium per unit of variance risk) is another important concept related to risk aversion. If the investors are becoming more risk averse over time, this will be reflected in the higher market price of risk. Similarly, if the investors are becoming less risk averse they will demand less risk premium per unit of risk and thus the market price of risk will decrease over time. However, if the risk aversion is constant over time, the market price of risk will remain constant over time. In this case, the variation in the excess return will be explained mostly by the variation in the market (variance) risk. Therefore, it will be useful to understand the time series behavior of market price of risk over time.

In order to empirically measure the investors' risk aversion, we need to use some existing finance theories that will help us choose variable or variables that can be used to measure the risk aversion. Tobin (1958) and Pratt (1964) are some of the earliest economists to link the market risk premium to the investor's risk aversion. In a continuous-time model, Merton (1973) has shown that risk premium of the same form as in the single-period model holds when the investment opportunity set is constant and portfolios are continuously rebalanced. For this reason and simplicity, the static CAPM is used in the empirical measurement of risk aversion (see Friend and Blume, 1975; French, Schwert, and Stambaugh, 1987; Chou, 1988; and Chou, Engle, and Kane, 1992).

When using the static CAPM, the risk aversion parameter can be obtained by dividing the expected excess return by the variance of the return on the stock market portfolio. Therefore, the estimation of the risk aversion is directly associated with the estimation of expected value and the variance of excess return on the market portfolio. One natural way to estimate the risk aversion is to use the ex post sample mean and sample variance (see Friend and Blume, 1975). As pointed out by Chou, Engle, and Kane (1992) (here after referred to as CEK), this is equivalent to using unconditional variance, which is not consistent with the assumption of the portfolio re-balancing. The risk premium should be determined by the conditional variance. Furthermore, the use of sample variance implicitly assumes that the variance does not change over time. However, the time-varying volatility of returns, or risk, in the stock market is well documented in the finance literature See for example, French, Schwert, and Stambaugh (1987).

Many studies use Autoregressive Conditional Heteroscedasticity (ARCH), Generalized 
ARCH (GARCH), or GARCH-in-mean (GARCH-M) models to model the behavior of conditional volatility (For a review, see Bollerslev, Chou, and Kroner (1992). The advantage of these models is that they allow the conditional variance $\left(h_{t}\right)$ to vary over time. Therefore, such models are suitable for the estimation of the risk aversion parameter.

Different versions of GARCH-M model, proposed by Engle, Lilien, and Robins (1987), can explicitly incorporate the risk aversion. These models are suitable for the estimation of risk aversion because these models use conditional variance instead of unconditional variance. However, these models implicitly assume the risk aversion parameter to be constant over time. CEK (Chou, Engle, and Kane, 1992) note that risk aversion of investors can change over time due to the changing preferences toward risk and/or changing investment opportunities.

One way to allow the risk parameter to change is to divide the whole sample into sub-samples and estimate the risk aversion parameter for each sub-sample using either the ratio formula or the GARCH-M model. Although, such method might give an idea about the changing behavior of the risk parameter, the partitioning of the whole sample into sub-samples can be arbitrary. Alternatively, one can use a rolling sample estimation process to estimate the time series of risk aversion parameter. However, due to the overlap of information from one sample to another, the estimate of the risk aversion parameter for a given period will be close to the risk aversion parameters for the immediately following and succeeding periods.

A more flexible approach will be to use the Time-Varying Parameter (TVP) GARCH-M model suggested by CEK. This model has some important attractive features. Firstly, it uses conditional variances rather than the unconditional variances. Secondly, the model allows the conditional variances to change over time conditional on the past information. Finally, it represents the risk parameter over time as a time series process. This allows for the estimation of risk aversion parameter over time without having to partition the sample into smaller samples. Therefore, as far as the estimation of the risk aversion is concerned, TVP GARCH-M model is the most suitable compared to the ratio formula and GARCH-M model.

In this paper, weekly excess return on Japanese stock market covering 2/7/1973 to $12 / 27 / 2000$ is used to estimate the risk aversion. The model used in this paper is the time-varying parameter (TVP) GARCH-M model proposed by CEK.

The remainder of the paper is organized as follows. In the next section, the model used in the paper is briefly described. In section 3, empirical results are presented and discussed. Conclusions are presented in the final section.

\section{The Model}

Let $y_{t}$ denote the excess return on the stock market portfolio. We can estimate the risk aversion parameter using unconditional mean and variance as follows:

$$
\text { Risk Aversion }=\frac{\bar{y}}{S_{y}^{2}}
$$


where $\bar{y}$ is the sample mean of the excess return $\left(y_{t}\right)$ and $S_{y}^{2}$ is the sample variance of the excess return. The formula given by equation (1) will be referred to as ratio formula. This is the method used by Friend and Blume (1975). A problem associated with their method is that we can obtain only one estimate of the risk aversion parameter per sample. This approach, therefore, essentially assumes that the risk aversion is constant for the entire sample period. One way to allow the risk aversion parameter to change over time would be to subdivide the sample into many sub-samples and estimate the risk aversion parameter for each sub-sample. However, such method is not satisfactory because there is a tradeoff between the sample size and number of sub-samples. If we divide the whole sample into many sub-samples this will lead to smaller sample size for each sub-sample this will lead to inefficient estimates of mean excess return and variance of excess return. Furthermore, this method may lead to negative value for the risk aversion parameter due to the fact that for some of the sub-sample, the likelihood that the mean excess return is negative would be higher smaller the sample size.

Alternatively, we can use sliding window to estimate the time series of risk-aversion parameter. However, such method is not satisfactory due to the fact that there will be too much overlapping between adjoining sub-samples therefore the risk aversion parameter would not change that much from window to window. Finally, both sub-sampling and sliding window methods use unconditional statistics, which is not appropriate as explained earlier.

The estimation of risk aversion parameter using the ratio formula in the sub-sample or sliding window can be termed as unconditional method because the variance is assumed to be constant throughout the sub-sample. The conditional method used in the estimation of risk aversion is the GARCH-M model Engle, Lilien, and Robins (1987). The model can be expressed as follows:

$$
\begin{gathered}
y_{t}=\gamma h_{t}+e_{t} \\
h_{t}=\alpha_{0}+\alpha_{1} e_{t-1}^{2}+\beta_{1} h_{t-1}
\end{gathered}
$$

where $\gamma$ is the risk aversion parameter and $e_{t}$ is the independently, identically and normally distributed random variable with conditional variance $h_{t}$. Equation (2) is known as the mean equation and equation (3) is known as the variance equation. This is a conditional model, which allows for the variance to change with time. It is important to note that even if the model is a conditional model and allows the variance to change over time (within the sample), it still assumes that the risk aversion parameter $\gamma$ to be constant within the sample.

The time varying parameter (TVP) GARCH-M model is a conditional model that allows the risk aversion parameter to change over time. The TVP GARCH-M model is presented below.

$$
\begin{aligned}
& y_{t}=\gamma_{t} h_{t}+e_{t} \\
& \gamma_{t}=\gamma_{t-1}+v_{t} \\
& h_{t}=\varphi_{0}^{2}+\phi_{1}^{2} e_{t-1}^{2}+\phi_{2}^{2} h_{t-1}
\end{aligned}
$$

where $v_{t}$ is a zero-mean serially independent and identically distributed normal random 
processes with variance $\sigma_{v}^{2}$. The stochastic processes $e_{t}$ and $v_{t}$ are assumed to be independent. Here the risk aversion parameter is assumed to follow a random walk process. Furthermore, the variance equation (6) is same as the variance equation (3) except the fact that the parameters in equation (6) have been squared in order to avoid negative value for the variance that would cause the numeric error during the numerical optimization. Note that if $\sigma_{v}^{2}=0$, then equation (3) would be the same as equation (2) and the whole system of equations will lead to the standard GARCH-M model. The unknown parameters of the model can be estimated using the Kalman filtering technique. The estimation technique used in the paper is based on the method suggested by Chou, Engle and Kane (1992) and Harvey, Ruiz and Sentana (1992). ${ }^{1}$

\section{Empirical Results}

In this paper, we use Datastream index for Japan is used to represent the stock market index for Japan. The weekly total return index for the period $2 / 7 / 1973$ to $12 / 27 / 2000$ is used to compute the excess return. The discount rate is used to represent the risk-free interest rate. The data set is obtained from the Datastream. The total return index and the weekly excess return are plotted in Figure 1 and Figure 2 respectively.

Before carrying out any of the conventional estimation of moments as well as the estimation of the TVP GARCH-M model, we must test to see if the excess return processes are stationary. If the excess return process were non-stationary, i.e., unit-root, then the conventional estimation of moments, like mean and variance would be invalid. Furthermore, the estimation procedure used to estimate the TVP GARCH-M models would also be invalid.

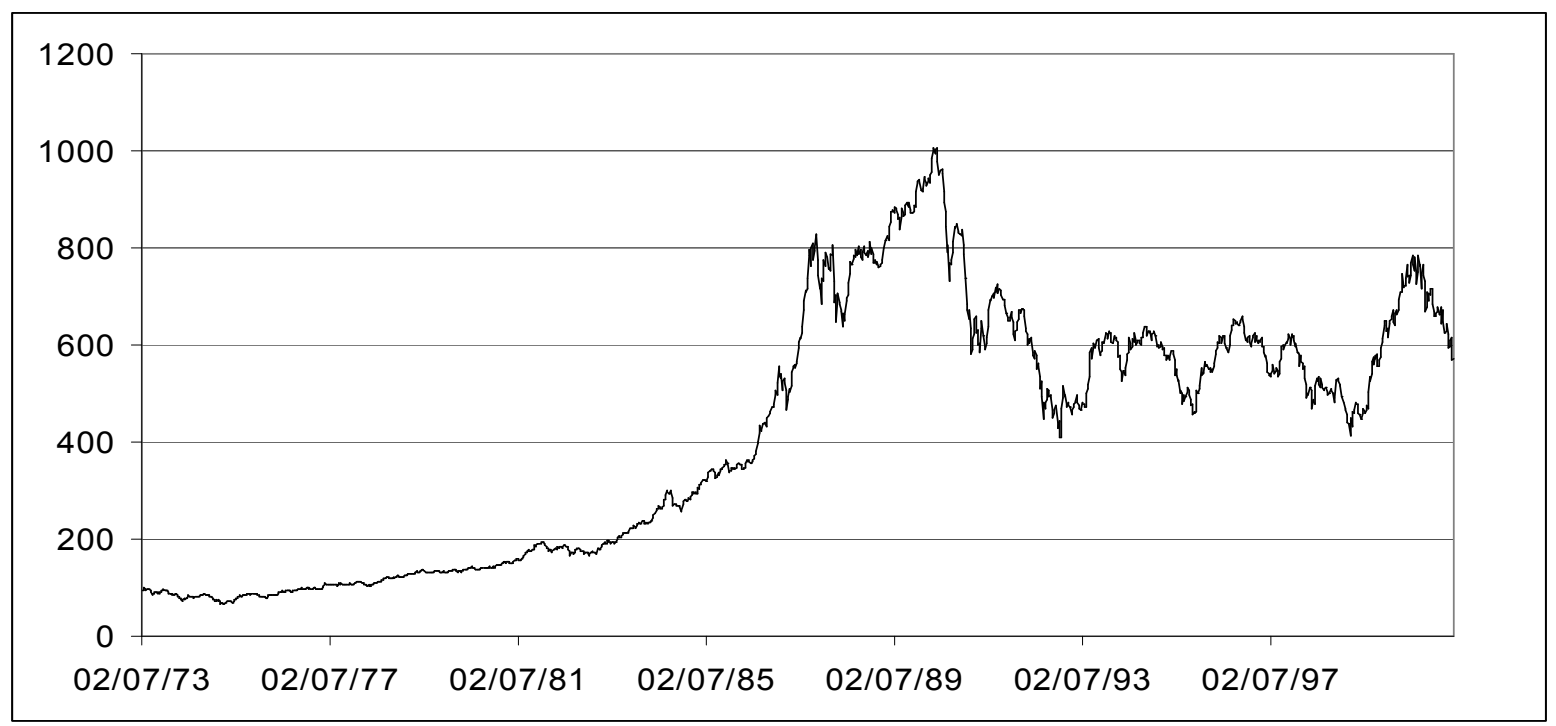

Figure 1: Plot of Total Return Data Stream Index for Japan (Weekly Data for 2/7/1973 12/27/2000)

1 Since both $h_{t}$ and $\gamma_{t}$ are unobservable $e_{t-1}^{2}$ is replaced by $\eta_{t-1}^{2}$ in equation (3) to estimate the parameters of the model. Following Chou, Engle, and Kane (1992), the innovation $\eta_{t}$ is defined as $\eta_{t}=y_{t}-E_{t-1}\left[\gamma_{t}\right] h_{t}$. 


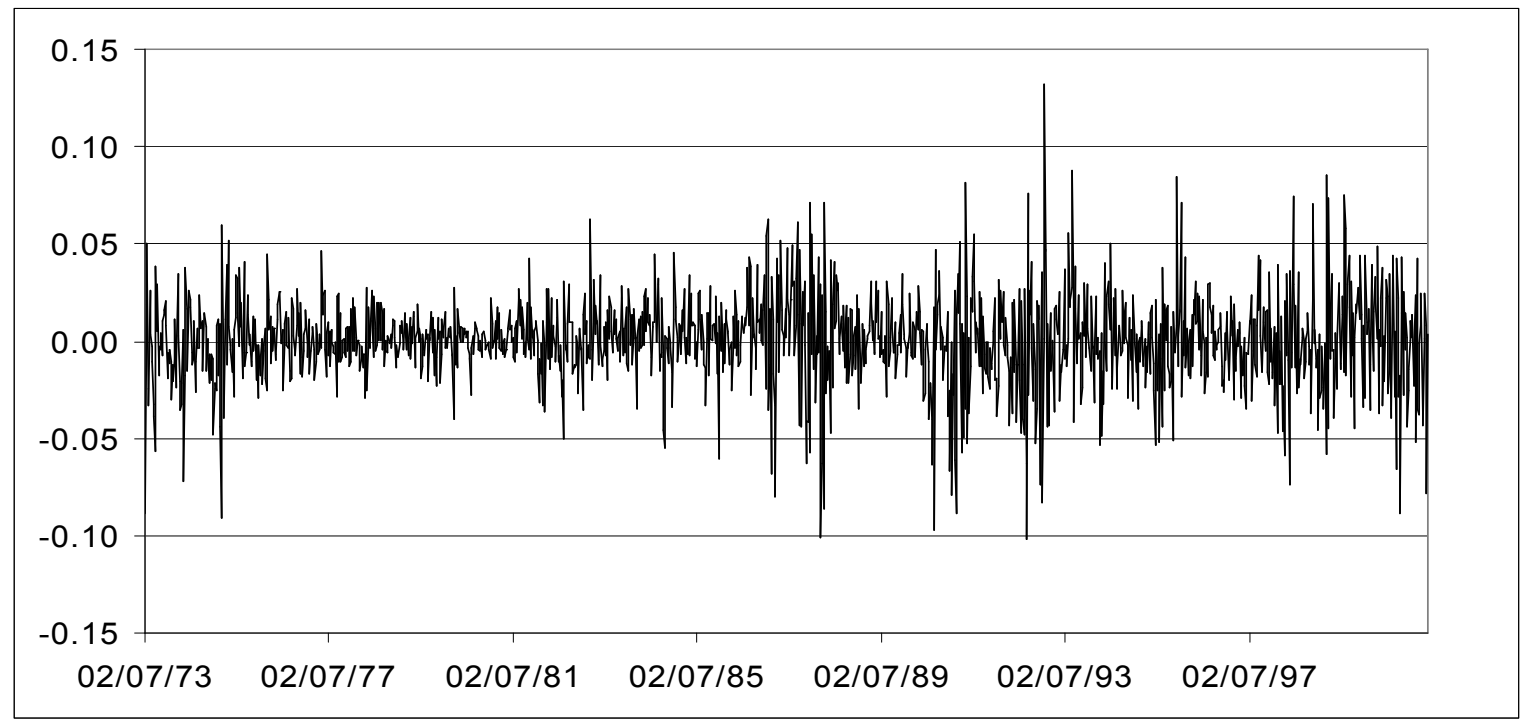

Figure 2: Plot of Weekly Excess Return for the Period (2/7/1973 - 12/27/2000)

The non-stationarity of the process can be tested using conventional Augmented Dickey-Fuller (see Dickey and Fuller $(1979,1981)$ ) or Phillips-Perron (see Phillips and Perron (1988)) unit root tests which are briefly described below.

For any given series $y_{t}$, the existence of unit root can be tested using the following Augmented Dickey-Fuller (ADF) regression equation

$$
\Delta y_{t}=\delta_{0}+\delta_{1} y_{t-1}+\sum_{j=1}^{p} \theta_{j} \Delta y_{t-j}+\varepsilon_{t}
$$

The null hypothesis of unit-root is given by $\delta_{1}=0$. The unit root (as a null hypothesis) can be tested using the usual t-statistic. The critical value can be found in Fuller (1976, p 371). The critical value at $1 \%$ is -3.51 The Phillips-Perron (PP) test also uses the t-statistic from equations (4) and (5). The difference is that PP corrects for the residual serial correlation. The ADF and PP test statistics are -38.446 and -38.609 respectively. Since both the test statistics are highly significant, we conclude that the excess return series is stationary. 
Table 1: Average Excess Return, Variance of Excess Return and Risk Aversion Parameter*

\begin{tabular}{|l|r|r|r|}
\hline \multicolumn{1}{|c|}{ Period } & $\begin{array}{c}\text { Average } \\
\text { Excess Return }\end{array}$ & $\begin{array}{c}\text { Variance of } \\
\text { Excess Return }\end{array}$ & $\begin{array}{c}\text { Risk Aversion } \\
\text { Parameter }\end{array}$ \\
\hline Whole $(2 / 7 / 1973-12 / 27 / 2000)$ & 0.00042 & 0.00054 & $\mathbf{0 . 7 8 5 8}$ \\
\hline 70 s $(2 / 7 / 1973-12 / 26 / 1979)$ & -0.00030 & 0.00033 & $\mathbf{- 0 . 9 3 4 0}$ \\
\hline 80 s $(1 / 2 / 1980-12 / 27 / 1989)$ & 0.00290 & 0.00041 & $\mathbf{7 . 0 3 6 4}$ \\
\hline 90s $(1 / 3 / 1990-12 / 29 / 1999)$ & -0.00094 & 0.00076 & $\mathbf{- 1 . 2 3 8 6}$ \\
\hline
\end{tabular}

*: The risk aversion parameter is estimated using the ratio of average excess return divided by variance of excess return.

The average excess return its variance and the risk aversion parameter computed using the ratio formula are given in Table 1 . For the whole sample period (2/7/1973 to $12 / 27 / 2000)$, the risk aversion parameter is estimated to be equal to 0.7858 . The risk aversion parameter is also computed for the 1970s, 1980s and 1990s. For 1970s and 1990s, the risk aversion parameters are negative and for $1980 \mathrm{~s}$ the risk aversion parameter is 7.0364 . The negative risk aversion parameter does not make sense and this is the result of subsampling as mentioned earlier.

In Table 2, we compute the time series of risk aversion parameter using the ratio formula and sliding window of length 52 weeks ( 1 year) and 104 weeks ( 2 years). The time series of risk aversion is also plotted in figure 3 .

Table 2: Summary Statistics of Risk Aversion Parameter Computed by Using the Ratio Formula.

\begin{tabular}{|l|c|c|c|}
\hline $\begin{array}{l}\text { Sliding Window } \\
\text { Length }\end{array}$ & $\begin{array}{c}\text { Average Risk } \\
\text { Aversion Parameter }\end{array}$ & $\begin{array}{c}\text { Maximum Risk } \\
\text { Aversion Parameter }\end{array}$ & $\begin{array}{c}\text { Minimum Risk } \\
\text { Aversion Parameter }\end{array}$ \\
\hline 52-Week Window & 4.2669 & 47.6923 & -16.8306 \\
\hline 104-Week Window & 3.4507 & 20.8350 & -6.9582 \\
\hline
\end{tabular}

As expected, the 104-week sliding window estimates of risk aversion parameter changes slower compared to the 52-week estimates. However, both estimates go down to the negative values. As pointed out earlier, this is the result of using ratio formula. 


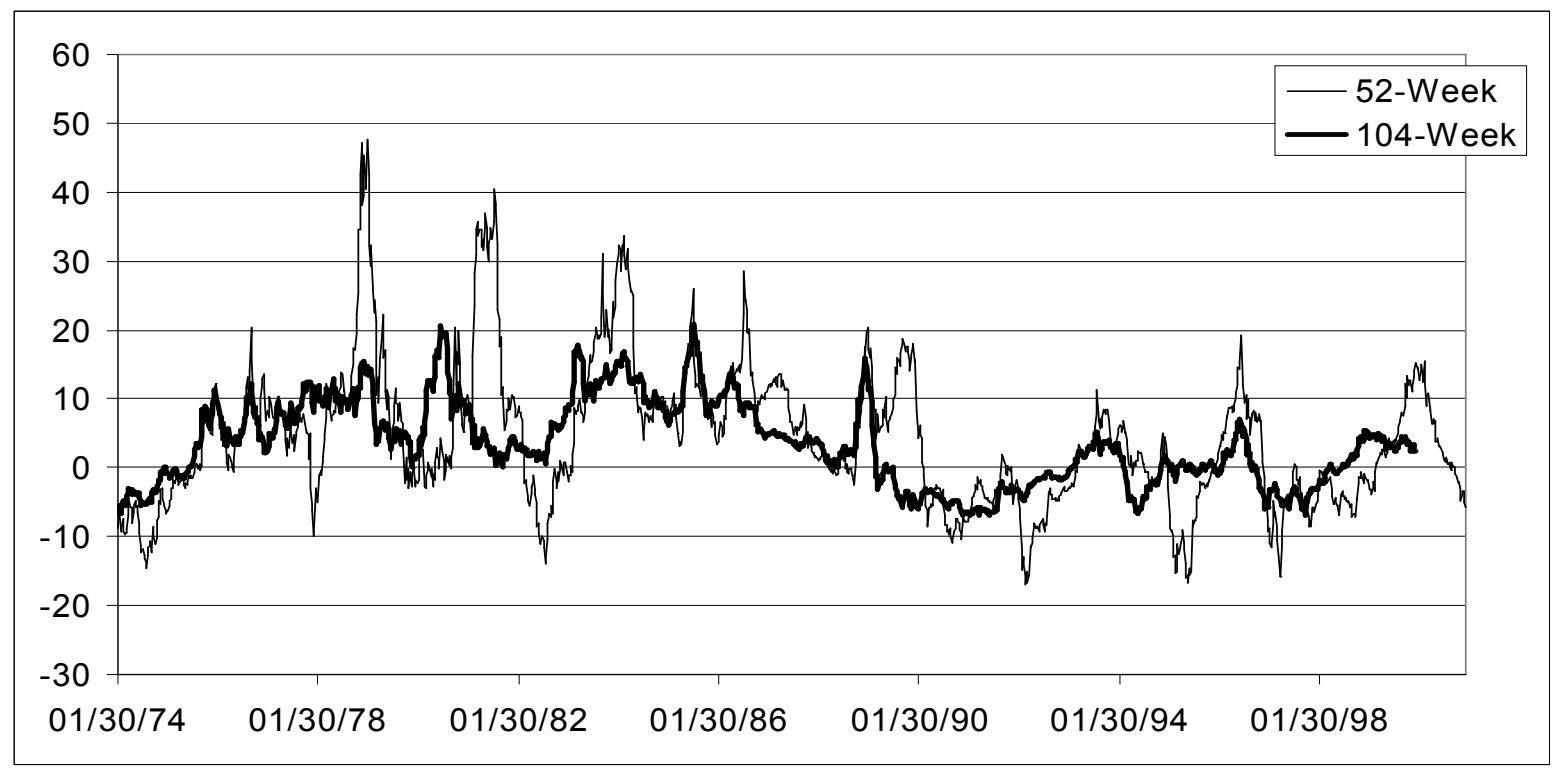

Figure 3: Plot of 52-Week and 102-Week Moving Window Estimate of Risk Aversion computed using Ratio Formula.

We also estimated the standard GARCH-M model. The results are summarized in Table 3. As it is clear from the table, all the parameters came out to be significant. The estimate of constant risk aversion parameter is 2.6576. As noted earlier, this model assumes the risk aversion parameter to be constant through the sample even if it allows the variance of the excess return to change over time. Therefore, this model cannot be used to estimate the time series of the risk aversion.

Table 3: Estimation of GARCH-M Model.

\begin{tabular}{|l|c|c|}
\hline & Coefficient & T-Value \\
\hline$\gamma$ & 2.6576 & 2.3632 \\
\hline$\alpha_{0}$ & $9.66 \mathrm{E}-06$ & 4.1211 \\
\hline$\alpha_{1}$ & 0.1144 & 8.5308 \\
\hline$\beta_{1}$ & 0.8723 & 60.9853 \\
\hline
\end{tabular}

In order to estimate the time series of risk aversion parameter, we need to use a more flexible model like the Time Varying Parameter (TVP) GARCH-M model proposed by Chou, Engle and Kane (1992) as discussed before. The TVP GARCH-M model is estimated using Kalman filtering technique. The result of the estimation is presented in Table 4. 
Table 4: Estimation of Time Varying Parameter GARCH-M Model.

\begin{tabular}{|c|l|c|}
\hline Parameter & Coefficient & T-Value \\
\hline$\sigma_{v}$ & 0.0256 & 0.565 \\
\hline$\phi_{0}$ & 0.0031 & 8.267 \\
\hline$\phi_{1}$ & 0.3385 & 17.054 \\
\hline$\phi_{2}$ & 0.9335 & 121.319 \\
\hline$\gamma_{0}$ & 3.0753 & 2.129 \\
\hline
\end{tabular}

The estimation of the time series of the risk aversion parameter is plotted in Figure 4 and the estimated volatility is plotted in Figure 5. The time series of the estimated risk aversion parameter is always positive. This is different from the time series of risk aversion parameter estimated using the sliding window, which is plotted in Figure 3. The sliding window estimate leads to many negative risk aversion parameters which do not make sense. TVP GARCH-M model takes care of this problem. The starting value of the risk aversion parameter $\left(\gamma_{0}\right)$ is 3.0753 and it increases to 3.463 in the middle of June 1987. There after, it starts a down ward trend and reaches around 2.2 by the end of 2000 .

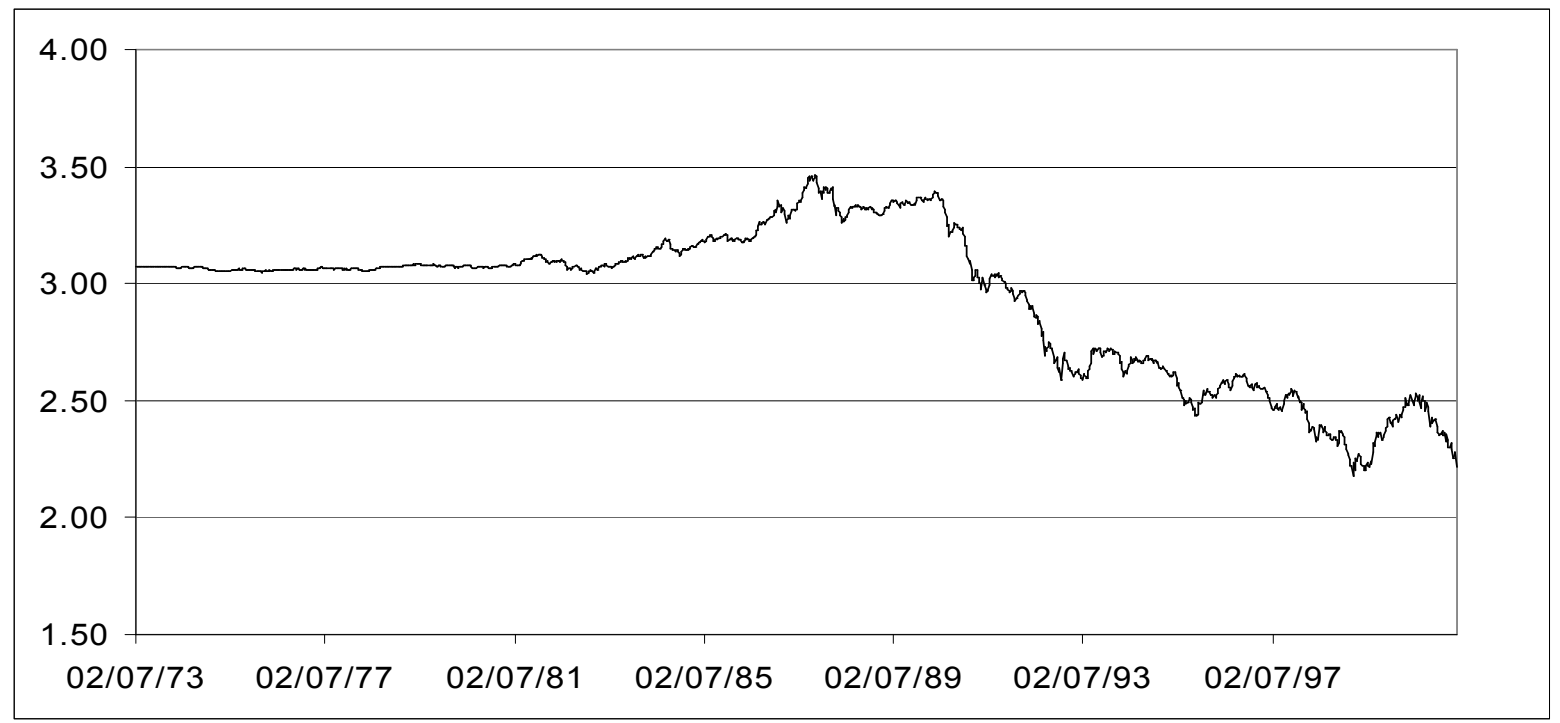


Figure 4: Plot of Risk Aversion Parameter computed using Time Varying Parameter Model.

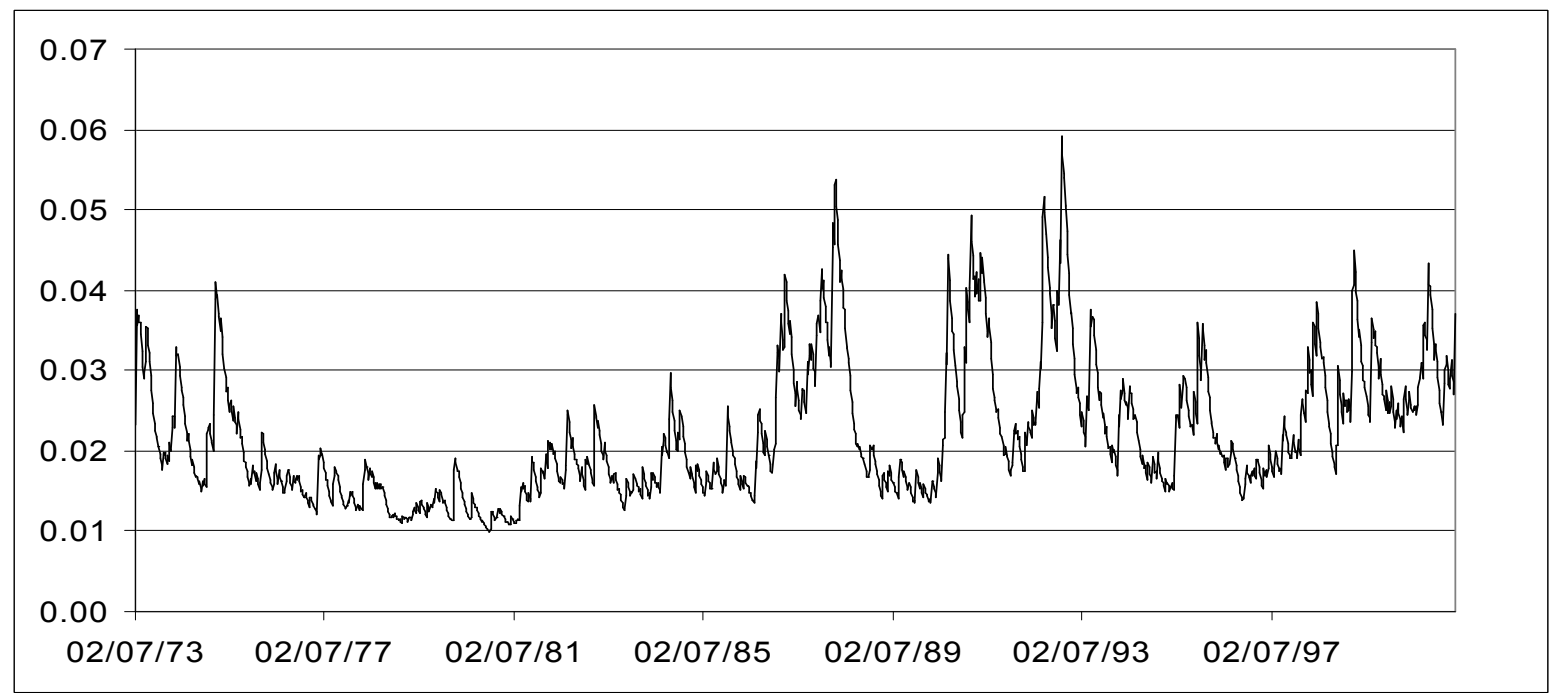

Figure 5: Conditional Volatility of the Excess Return $\left(\sqrt{h_{t}}\right)$.

Since the estimate of $\sigma_{v}$ is not significantly different from zero, it looks that the risk aversion parameter has not significantly changed in last three decades in Japan. This indicates that most of the variation in excess return over the period under consideration is explained by the changes in market (variance) risk instead of changes in risk aversion itself.

\section{Conclusions}

In this paper, the time series of risk aversion parameter is estimated for Japan. We used weekly data covering $2 / 7 / 1973$ to $12 / 27 / 2000$. The risk aversion parameter, estimated using the ratio of average excess return to the variance of the excess return, for the whole period is 0.7858. When the sample is divided into three sub-samples, the estimate of the risk aversion parameters are $-0.9340,7.0364$ and -1.2386 respectively for the $1970 \mathrm{~s}, 1980 \mathrm{~s}$ and $1990 \mathrm{~s}$.

There are two basic problems associated with the use of the ratio formula in the estimation of the risk aversion parameter. Firstly, the method assumes that for the sample period the risk aversion parameter as well as the sample moments is constant. Secondly, it may lead to negative risk aversion parameter which is not meaningful. The second model which is a conditional model and which allows the variance to change over time is the GARCH-M model. Using the GARCH-M model, we estimate the risk aversion parameter for the whole sample period to be equal to 2.657. Even if the model allows for the variance to change over time, it still assumes the risk aversion parameter to be constant through the sample period.

Therefore, we use Time Varying Parameter (EVP) GARCH-M model suggested by Chou, Engle and Kane (1992), which allows for the risk aversion parameter to change over time by modeling the risk aversion parameter to follow a random walk process. The use of the TVP 
GARCH-M model eliminated the problem of negative risk aversion. It also allowed us to estimate the time series of risk aversion parameter. The risk aversion parameter range is between 3.5 to 2.2. We also find that the risk aversion parameter has not significantly changed over time. This implies that most of the variation in excess return can be explained by the variation in the market (variance) risk.

\section{References}

Baillie, Richard T and Tim Bollerslev, 1994, Cointegration, Fractional cointegration and Exchange Rate Dynamics, Journal of Finance, Vol. 49, pp. 737-745.

Bollerslev, T., R. Chou, and K. Kroner, 1992, ARCH Modeling in Finance: A Review of the Theory and Empirical Evidence, Journal of Econometrics, Vol. 52, pp. 5-59.

Chou, R.Y. (1988), Volatility Persistence and Stock Valuations, Journal of Applied Econometrics, Vol. 3, pp. 279-294.

Chou, R., R. F. Engle, and A. Kane, 1992, Measuring Risk Aversion from Excess Returns on a Stock Index, Journal of Econometrics, Vol. 52, pp. 201-224

Dickey, David and Wayne A. Fuller, 1979, Distribution of the Estimates for Autoregressive Time Series with a Unit Root, Journal of the American Statistical Association, Vol. 74, pp. $427-431$

, 1981, Likelihood Ratio Statistics for Autoregressive Time Series with a Unit Root, Econometrics, Vol. 49, pp 1057-1072.

French, K. R., G. Schwert, and R. Stambaugh, 1987, Expected Stock Returns and Volatility, Journal of Financial Economics, Vol. 19, pp.3-30

Engle, R.F., D. Lilien and R. Robins, 1987, Estimating Time Varying Risk Premia in the Term Structure: The ARCH-M model, Econometrica, Vol. 55, pp. 391-407.

Friend, I. And M. Blume, 1975, The Demand for Risky Assets, American Economic Review, Vol. 65, pp. 900-922.

Harvey, Andrew, Esther Ruiz and Enrique Sentana, 1992, Unovserved Component Time Series Models with ARCH Disturbances, Journal of Econometrics, Vol. 52, pp. 129-157.

Merton, R.C. , 1973, An Intertemporal Capital Asset Pricing Model, Econometrica, Vol. 41, pp. 867-887.

Pratt, J.W., 1964, Risk Aversion in the Small and in the Large, Econometrica, Vol. 32, pp. 122-136.

Tobin, J., 1958, Liquidity Preference as Behavior toward Risk, Review of Economics Studies, Vol. 26, pp. 65-86. 